\title{
Sevoflurane anesthesia persistently downregulates muscle-specific microRNAs in rat plasma
}

\author{
JUMPEI TAKEUCHI $^{1}$, ATSUHIRO SAKAMOTO ${ }^{1}$ and TOSHIHIRO TAKIZAWA ${ }^{2}$ \\ Departments of ${ }^{1}$ Anesthesiology and Pain Medicine, ${ }^{2}$ Molecular Medicine and Anatomy, \\ Graduate School of Medicine, Nippon Medical School, Tokyo 113-8602, Japan
}

Received December 2, 2013; Accepted March 31, 2014

DOI: $10.3892 /$ ijmm.2014.1739

\begin{abstract}
The volatile anesthetic, sevoflurane, is widely used in surgery. Over the years, there has been a growing interest in the biological effects of sevoflurane on tissue and organ systems and the molecular mechanisms involved. MicroRNAs (miRNAs or miRs) acting as pivotal post-transcriptional regulators for fine-tuning gene networks are not only expressed intracellularly, but are also secreted into the plasma. However, the sevoflurane-associated dynamics of circulating miRNAs and the effects of sevoflurane on tissues remain unknown. Thus, the aim of this study was to perform a comprehensive analysis of circulating miRNA levels and compositions in sevoflurane-anesthetized rats. The rats were allowed to breathe spontaneously under $2 \%$ sevoflurane anesthesia for $6 \mathrm{~h}$, and we performed a quantitative polymerase chain reaction (PCR)-based array analysis of the time-dependent changes in plasma miRNA levels and compositions. Subsequently, we validated the levels of muscle-specific miRNAs (also known as myomiRNAs; $m i R-1, m i R-133 a, m i R-133 b$ and $m i R-206$ ) of the plasma, heart and skeletal muscle by quantitative PCR following 3 and $6 \mathrm{~h}$ of anesthesia, as well as at 1, 3, 7 and 14 days post-anesthesia. Of the 210 miRNAs detected in the rat plasma from the control group (no anesthesia), 161 plasma miRNAs (77\%) were transiently downregulated as a result of sevoflurane anesthesia. Although the downregulation of the plasma miRNAs (148 out of the 161 plasma mRNAs; 92\%) recovered immediately after anesthesia, the plasma levels of 4 muscle-specific miRNAs were persistently downregulated until 14 days post-anesthesia. In the cardiac and skeletal muscles, the expression levels of the muscle-specific miRNAs were upregulated within 2 weeks post-anesthesia, indicating that the expression levels of the muscle-specific miRNAs in
\end{abstract}

Correspondence to: Professor Toshihiro Takizawa, Department of Molecular Medicine and Anatomy, Graduate School of Medicine, Nippon Medical School, 1-1-5 Sendagi, Tokyo 113-8602, Japan E-mail: t-takizawa@nms.ac.jp

Key words: anesthesia, sevoflurane, circulating microRNA, musclespecific microRNA, cardioprotection, rat the cardiac and skeletal muscles and their plasma levels are substantially inversely correlated following anesthesia. Our data suggest that sevoflurane predominantly affects cardiac and skeletal muscles and suppresses the release of miRNA from these tissues into the circulation. This new information provides novel insight into the molecular mechanisms of action of the anesthetic, sevoflurane.

\section{Introduction}

The volatile anesthetic, sevoflurane, is widely used in surgery as there is extensive clinical data supporting its safety (1). Over the years has been a growing interest in the biological effects of sevoflurane on tissue and organ systems and the molecular mechanisms involved. The heart and the brain are the major target organs of sevoflurane; sevoflurane can protect the heart against ischemic damage (2). Several signal transduction pathways, such as the activation of $\mathrm{G}$ protein-coupled receptors, protein kinase C (PKC), phosphoinositide 3-kinase (PI3K), extracellular signal-regulated kinase (Erk)1/2 and mitochondrial KATP channels, have been implicated in the molecular mechanisms of myocardial protection by volatile anesthetics, including sevoflurane (3). However, the cellular and molecular processes by which sevoflurane exerts protective effects on the heart are incompletely understood.

MicroRNAs (miRNAs or miRs), small, non-coding RNAs of approximately 22 nucleotides (nt) in length, play a critical role in the post-transcriptional regulation of their target genes at the mRNA and/or protein level (4). Dysregulated miRNA expression has been implicated in a number of pathophysiological mechanisms, such as oncogenesis (5) and cardiovascular disease (6). Moreover, miRNAs are not only localized intracellularly, but are also secreted into extracellular fluid, such as plasma, serum, saliva and urine, via exosomes, i.e., tiny vesicles of 50-100 nm in diameter (7). Exosomal miRNAs are generally considered to be stable in the circulation (8). This has raised the possibility that circulating miRNAs may serve as novel biomarkers for detecting and monitoring various pathophysiological conditions, such as cancer (8).

Certain studies in the field of anesthesiology have been conducted to explore tissue- and organ-specific alterations in miRNA expression induced by volatile anesthetics in rats $(9,10)$. Tanaka et al (9) reported significant changes in miRNA expression profiles in rat lungs under sevoflurane anesthesia. Moreover, 
Ishikawa et al (10) demonstrated differential miRNA expression patterns between sevoflurane and propofol anesthesia in the rat liver. Based on the findings regarding circulating miRNAs, plasma miRNAs may be informative markers for monitoring and assessing the effects of the volatile anesthetic modification of miRNA expression in organs and body systems. However, volatile anesthetic-associated dynamics of circulating miRNAs and the detailed mechanisms of the effects of volatile anesthetics on tissues and organs remain unclear.

Thus, the aim of this study was to perform a comprehensive analysis of the time-dependent changes in circulating miRNA levels and composition in sevoflurane-anesthetized rats using a quantitative polymerase chain reaction (PCR)-based array. We discovered that the persistent downregulation of muscle specific-miRNAs (also known as myomiRNAs) occurred for 2 weeks following sevoflurane anesthesia. Sevoflurane anesthesia may predominantly affect cardiac and skeletal muscles and suppress miRNA secretion from these tissues into the circulation.

\section{Materials and methods}

Sample preparation. This study was approved by the Animal Research Committee of Nippon Medical School, Tokyo, Japan. Six-week-old male Wistar rats (Saitama Experimental Animals Supply, Saitama, Japan), weighing 180 \pm 20 g, were maintained under a 12/12-h light/dark cycle in a temperature-controlled environment. Sevoflurane anesthesia was performed using the procedure employed in our previous miRNA studies on rats $(9,10)$. Briefly, each rat was allowed to breathe spontaneously, housed in an anesthesia box (width, $690 \mathrm{~mm}$; diameter, 410 mm; height, 330 mm; MAB-2; Sanplatec Corp., Osaka, Japan), and supplied with an air-oxygen mixture (fraction of inspired oxygen, 0.4 ) at a rate of $61 / \mathrm{min}$, with body temperature maintained at approximately $37^{\circ} \mathrm{C}$ using a heat lamp. Rats undergoing sevoflurane anesthesia were supplied in the box with $2.0 \%$ (1 'minimum alveolar concentration') sevoflurane (Maruishi Pharmaceutical Co., Ltd., Osaka, Japan) for $6 \mathrm{~h}$ (designated as 6-h anesthesia), as previously described (11); this dose is commonly used clinically. The control group rats received no anesthesia. There were no significant differences in physiological data during anesthesia between the anesthesia groups and the control group. Hypoxia, hyper/hypocapnia, hypotension, or hypothermia did not occur in any of the groups. The animals were sacrificed either immediately after cessation of the 6-h anesthesia, or after the recovery periods that lasted 1, 3, 7 and 14 days following the termination of exposure (referred to as days 1, 3, 7 and 14 post-anesthesia, respectively).

In the initial PCR-based array, the rats were assigned to 4 groups ( $n=6$ per group): i) the control group (no anesthesia), ii) the 6 -h anesthesia group, iii) the day 1 post-anesthesia group and iv) the day 7 post-anesthesia group. In all the groups, rat blood samples were obtained from the inferior vena cava within 3 min after sacrifice by cervical dislocation.

In a subsequent validation assay, the rats were assigned to 7 groups ( $n=6$ per group): i) the control group, ii) the 3 -h anesthesia group, iii) the 6 -h anesthesia group, iv) the day 1 post-anesthesia group, v) the day 3 post-anesthesia group, vi) the day 7 post-anesthesia group and vii) the day 14 post- anesthesia group. Samples [blood, heart and skeletal muscle (quadriceps femoris)] were obtained within $5 \mathrm{~min}$ after sacrifice by cervical dislocation.

RNA preparation. To separate blood plasma, blood samples were collected into EDTA vacuum blood collection tubes (Venogect II; Terumo, Tokyo, Japan) and then centrifuged $\left(1,700 \times \mathrm{g}, 15 \mathrm{~min}, 4^{\circ} \mathrm{C}\right)$. The collected plasma samples were transferred to RNase/DNase-free 1.5-ml microcentrifuge tubes, and stored at $-80^{\circ} \mathrm{C}$ before RNA purification. Total RNA from plasma and tissue samples was extracted using Isogen-LS and Isogen (both from Wako, Osaka, Japan), respectively, according to the manufacturer's instructions.

Comprehensive quantitative analysis of circulating miRNAs by quantitative PCR-based array. For the initial quantitative PCR-based array, equal quantities from each plasma sample were pooled within each group; total RNA were extracted from the pooled plasma samples as described above. Total RNA extracted from the equivalent of $25-\mu 1$ plasma was reversetranscribed using Megaplex RT Primers (Applied Biosystems, Foster City, CA, USA). The cDNA was then pre-amplified using Megaplex PreAmp Primers (Applied Biosystems). The pre-amplified products were subjected to quantitative PCR using TaqMan MicroRNA Assay Rodent Panels (A and B, v.3.0) on a 7900HT Fast Real-Time PCR System (Applied Biosystems) according to the manufacturer's instructions. miRNA sequences were annotated using the Sanger database (miRBase), release 14. Data obtained with this assay were analyzed using RQ Manager 1.2 (Applied Biosystems). For the quantification of each miRNA expression level, the comparative $\mathrm{Ct}$ method ( $\Delta \Delta \mathrm{Ct}$ method) was used as described below. Full array data sets are available upon request.

Quantitative analysis of miRNA expression by quantitative PCR. Quantitative PCR of the miRNAs was performed using TaqMan Gene Expression assays (Applied Biosystems) in a 7300 Real-Time PCR System (Applied Biosystems), according to the manufacturer's instructions. To normalize the miRNA expression levels, Caenorhabditis elegans miRNAs (cel-miRNAs) and Rnu6 were used as exogenous internal controls for the plasma samples and an endogenous internal control for the tissue samples, respectively.

Normalization of PCR data for circulating miRNAs using exogenous 'spike-in' cel-miRNAs. We used a fixed volume of RNA eluate $(2 \mu \mathrm{l})$ from a given volume of starting plasma as input for the RT reaction. For a sample in which the starting plasma volume was $125 \mu \mathrm{l}$, an input of $2 \mu \mathrm{l}$ of eluted RNA (taken from a total RNA eluate volume of $\sim 20 \mu \mathrm{l}$ ) into the $\mathrm{RT}$ reaction corresponds to the mass of RNA derived from $\sim 12.5 \mu \mathrm{l}$ of starting plasma.

Both PCR-based array and quantitative PCR data of the plasma miRNAs were normalized to exogenous cel-miRNAs as a 'spike-in' control using a modification of the method described in the study by Mitchell et al (8). Briefly, 2 synthetic RNA oligonucleotides corresponding to cel-miR-39 and cel-miR-238 (Qiagen, Valencia, CA, USA) were used. The 'spike-in' oligos were introduced (as a mixture of $25 \mathrm{fmol}$ of each oligonucleotide in 5- $\mu 1$ water) after the addition of 
A $\quad 6$-h anesthesia vs control group

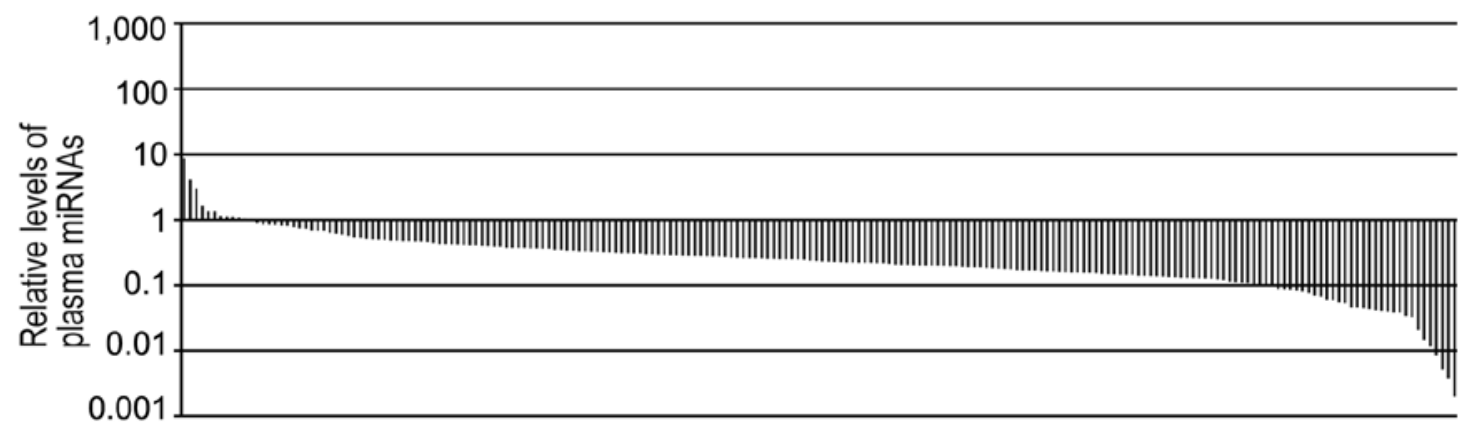

B Day 1 post-anesthesia vs control group

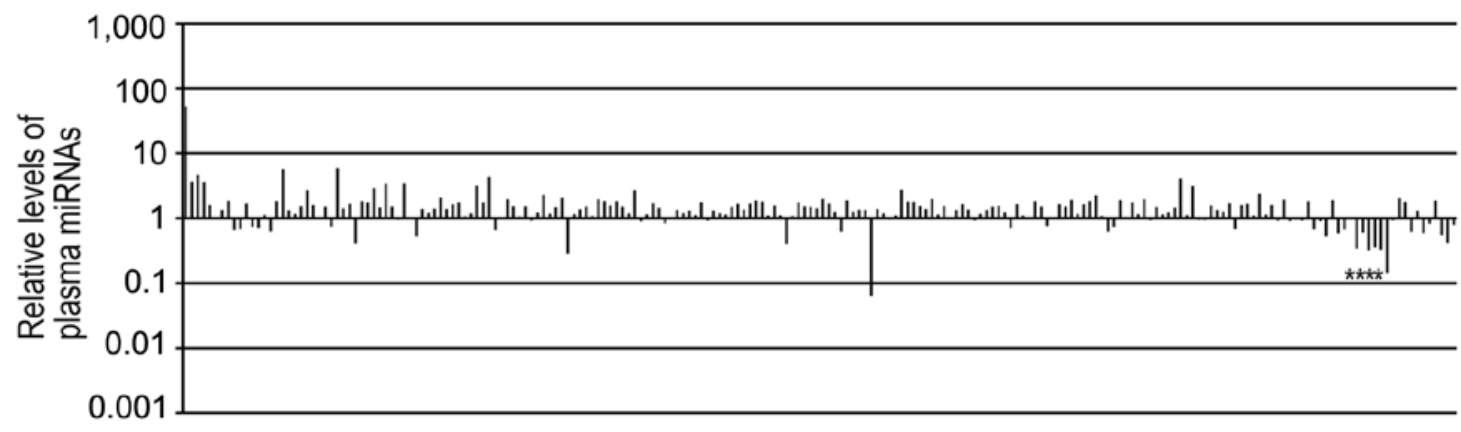

C Day 7 post-anesthesia vs control group

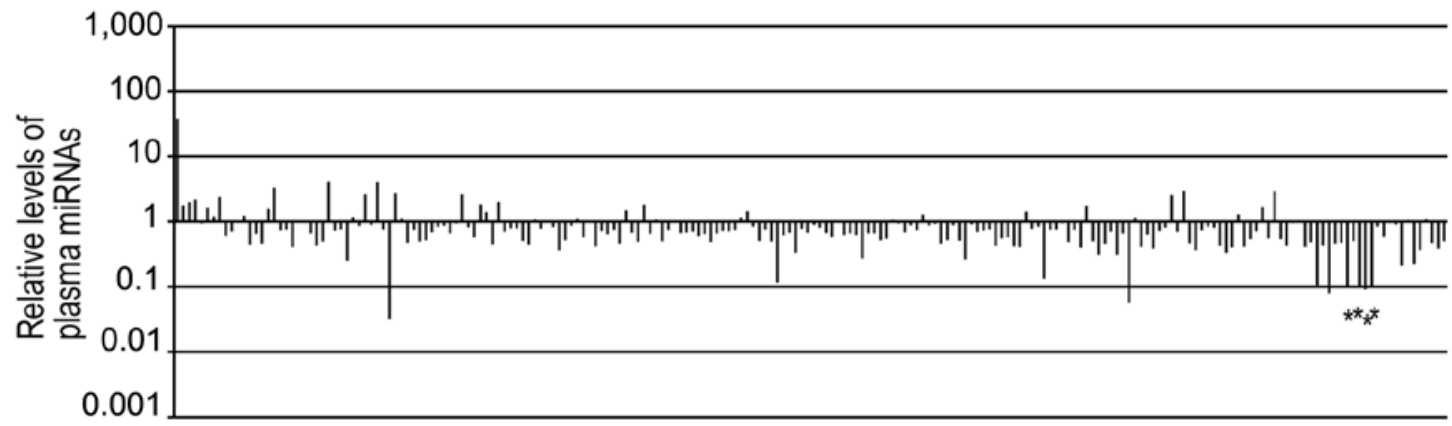

Figure 1. Plasma miRNA landscape in sevoflurane-anesthetized rats, as determined using a quantitative polymerase chain reaction (PCR)-based miRNA expression array. Differential profiles of plasma miRNAs in (A) the 6-h anesthesia group, (B) the day 1 post-anesthesia group, and (C) the day 7 post-anesthesia group in comparison with the control group (no anesthesia). The full data set is available upon request. Data were normalized using the exogenous Caenorhabditis elegans miRNAs (cel-miRNAs) (cel-miR-39 and cel-miR-238). The x-axis indicates the miRNA rank, from the most upregulated to the most downregulated miRNAs, based on the differential profile of 210 plasma miRNAs in the 6-h anesthesia group compared with the control group. The y-axis is the log fold change in plasma miRNA levels relative to the control group. Asterisks (*) indicate muscle-specific miRNAs ( $m i R-1, m i R-133 a, m i R-133 b$ and $m i R-206)$.

Isogen-LS to the plasma samples. For each RNA sample, the cel-miRNAs were measured using TaqMan qRT-PCR assays (Applied Biosystems) as described above. The threshold cycle $(\mathrm{Ct})$ values obtained for the 2 'spike-in' cel-miRNAs were averaged to generate a 'spike-in' control $\mathrm{Ct}$ value. This subsequently produced a different $(\Delta \mathrm{Ct})$ value for each plasma miRNA based on the following formula: $\Delta \mathrm{Ct}=$ (plasma miRNA Ct value of a given sample) - ('spike-in' control $\mathrm{Ct}$ value of the sample). A sample in the control group (no anesthesia) was arbitrarily designated as the calibrator sample (1X sample), and the relative miRNA levels of samples in all other groups were then expressed relative to the calibrator sample. Thus, the value of $\Delta \Delta \mathrm{Ct}$ for each testing sample was determined by the formula: $\Delta \Delta \mathrm{Ct}=\Delta \mathrm{Ct}$ (testing sample) $-\Delta \mathrm{Ct}$ (calibrator sample).

Statistical analysis. We conducted all analyses using SPSS software (v.20 for Windows; IBM-SPSS). The significance of between-group differences was assessed using ANOVA followed by Dunnett's test. P-values $<0.05$ were considered to indicate statistically significant differences. Values are expressed as the means \pm standard deviation (SD). 
Table I. Representative miRNAs significantly downregulated or upregulated in rat plasma in a quantitative PCR-based array.

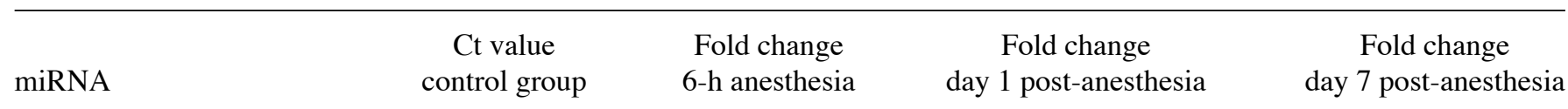

Downregulated miRNAs ${ }^{\mathrm{a}}$

$\begin{array}{lllll}\text { miR-133a } & 19.86 & 0.032 & 0.311 & 0.075 \\ m i R-206 & 22.61 & 0.046 & 0.329 & 0.225 \\ m i R-133 b & 22.89 & 0.033 & 0.333 & 0.072 \\ m i R-1 & 23.80 & 0.030 & 0.348 & 0.066 \\ m i R-433 & 25.60 & 0.243 & 0.273 & 0.265 \\ m i R-350 & 29.20 & 0.221 & 0.063 & 0.272\end{array}$

Upregulated miRNAs ${ }^{\mathrm{b}}$

miR-148b-5p

35.88

8.601

52.552

37.969

${ }^{a}$ miRNAs that were downregulated by $>2.5$-fold in both the day 1 and 7 post-anesthesia groups compared with the control group. ${ }^{b}$ miRNAs that were upregulated by $>2.5$-fold in both the day 1 and 7 post-anesthesia groups compared with the control group. miRNAs, microRNAs; PCR, polymerase chain reaction.

\section{Results}

Comprehensive profile analysis of circulating microRNAs using quantitative PCR-based miRNA expression array. Among the 373 preloaded rat miRNAs contained in realtime PCR-based miRNA expression profiling array cards, 210 miRNAs (56\% of the preloaded miRNAs) were detected in rat plasma from the control group (no anesthesia). Surprisingly, 161 plasma miRNAs (77\% of the miRNAs detected in the control group) were decreased by $>2.5$-fold in the 6 -h anesthesia group compared with the control group (Fig. 1A). By contrast, 46 plasma mRNA levels (22\%) remained unlatered (i.e., between 2.5-fold underexpressed and overexpressed), and only 3 miRNAs (1\%) were increased by $>2.5$-fold in the 6-h anesthesia group compared with the control group. Fold differences in miRNAs ranged from 0.002- to 8.6-fold change in the 6-h anesthesia group relative to the control group (median, 0.234). It should be noted that the majority of the miRNAs detected in the control group were downregulated in the rat plasma during sevoflurane anesthesia.

Following sevoflurane anesthesia, the levels of the plasma miRNAs which had decreased within $6 \mathrm{~h}$ of anesthesia, substantially increased in the day 1 post-anesthesia group: of the 161 plasma miRNAs decreased by $>2.5$-fold in the 6 -h anesthesia group compared with the control group, the levels of 148 of these miRNAs (92\%) returned to control group levels (between 2.5-fold underexpressed and overexpressed) (Fig. 1B). Fold differences in miRNAs ranged from 0.063- to 52.6-fold changes in the day 1 post-anesthesia group relative to the control group (median, 1.331). In the day 7 post-anesthesia group, of the 210 miRNAs detected in rat plasma from the control group, the levels of 176 of these miRNAs (84\%) were between 2.5 -fold underexpressed and overexpressed as compared with those of the control group (Fig. 1C). Fold differences in miRNAs ranged from 0.032 - to 38.0 -fold change in the day 7 postanesthesia group relative to the control group (median, 0.695). Only 6 miRNAs were downregulated by $>2.5$-fold in both the day 1 and day 7 post-anesthesia groups compared with the control group (Table I). Of note, 4 miRNAs (miR-1, miR-
$133 a$, miR-133b and $m i R-206)$, which have been reported to be muscle-specific miRNAs (12), were included in the 6 plasma miRNAs that were persistently downregulated following sevoflurane anesthesia. In terms of persistently upregulated miRNAs, only 1 miRNA, miR-148b-5p, was upregulated by $>2.5$-fold in both the day 1 and day 7 post-anesthesia groups compared with the normal (control) group (Table I).

Although many miRNAs are expressed ubiquitously in mammals, some miRNAs exhibit specific expression patterns in a tissue- or cell type-dependent manner (13). Several miRNAs, such as $m i R-1, m i R-133 a, m i R-133 b$ and $m i R-206$, are specifically expressed in striated muscle tissue. It seems reasonable that plasma muscle-specific miRNAs mirror an altered miRNA status in cardiac and skeletal muscle tissue. Thus, it may be that the decreased plasma levels of musclespecific miRNAs were associated with the sevoflurane-induced inhibition of the release of miRNAs from cardiac and skeletal muscle tissues. Additionally, considering the experimental and technical aspects of plasma miRNA application as potential biomarkers to evaluate the effects of volatile anesthetics, the 4 muscle-specific miRNAs detected at low $\mathrm{Ct}$ values in rat plasma may be advantageous in terms of sensitivity and reliability as a low $\mathrm{Ct}$ value corresponds to a high miRNA level in the plasma (Table I). Thus, we then focused on the muscle-specific miRNAs that were persistently downregulated following sevoflurane anesthesia.

Validation of dynamics of plasma muscle-specific miRNAs by quantitative PCR. To confirm whether plasma musclespecific miRNAs were indeed downregulated by sevoflurane, the plasma levels of 4 muscle-specific miRNAs (miR-1, $m i R-133 a, m i R-133 b$ and $m i R-206)$ were analyzed during and after sevoflurane anesthesia by quantitative PCR (Fig. 2). Quantitative PCR was performed at extended time points: at 3 and $6 \mathrm{~h}$ of anesthesia, and at 1,3, 7 and 14 days postanesthesia. The plasma levels of all 4 muscle-specific miRNAs decreased significantly at $3 \mathrm{~h}$ after the induction of anesthesia and were downregulated until 14 days post-sevoflurane anesthesia (Fig. 2). 

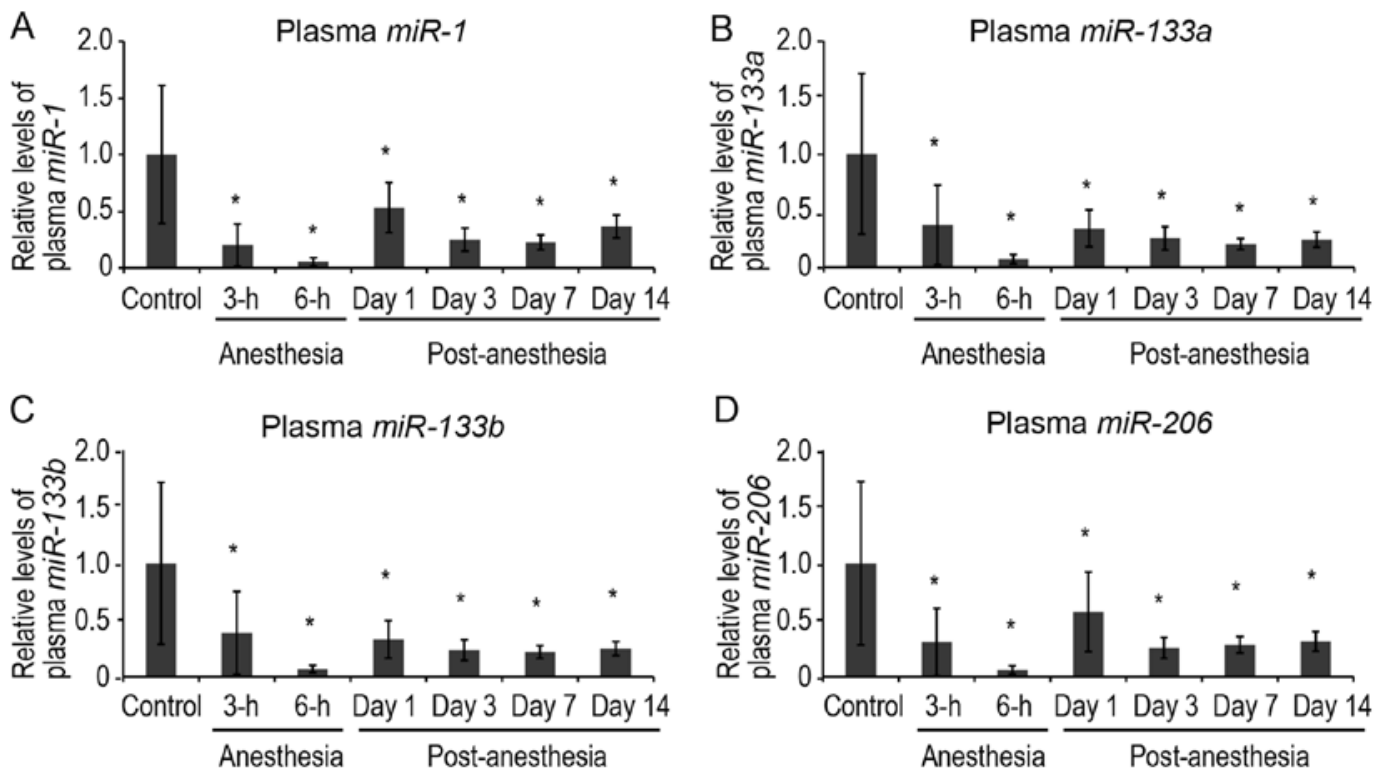

Figure 2. Validation of the dynamics of plasma muscle-specific miRNAs by quantitative polymerase chain reaction (PCR). The plasma levels of musclespecific miRNAs [(A) $m i R-1$, (B) $m i R-133 a$, (C) $m i R-133 b$ and (D) $m i R-206$ ] were confirmed by quantitative PCR. Time points for quantitative PCR analysis were: 3 - and 6-h anesthesia, and at 1,3,7 and 14 days post-anesthesia. Data are the means \pm standard deviation (SD) (n=6 per group). Data were normalized to Caenorhabditis elegans miRNAs (cel-miRNAs). Levels of miRNAs in the control group (no anesthesia) were defined as 1 . Dunnett's test; ${ }^{*} \mathrm{P}<0.05$.

Expression levels of muscle-specific miRNAs in cardiac and skeletal muscle. It is conceivable that the suppression of the release of miRNAs from cardiac and/or skeletal muscle tissue is the major cause of the decrease in plasma muscle-specific miRNA levels. To investigate this matter, we examined the expression levels of these miRNAs in cardiac and skeletal muscle ( $\mathrm{n}=6$ per group) (Figs. 3 and 4). $m i R-1$ and $m i R-133 a$ were expressed in both skeletal and cardiac muscle, while $m i R$ $133 \mathrm{~b}$ and $\mathrm{miR}$-206 were expressed solely in skeletal muscle, in accordance with a previous report (12).

In cardiac muscle, the expression levels of $m i R-1$ were increased significantly at 1 day post-anesthesia and were upregulated until 14 days post-anesthesia (Fig. 3). The expression levels of $m i R-133 a$ were also significantly increased at 7 and 14 days post-anesthesia. In the skeletal muscle, the expression levels of muscle-specific miRNAs tended to increase following sevoflurane anesthesia (Fig. 4). The expression levels of $m i R-1$ were increased significantly at 7 and 14 days post-anesthesia. A significant increase in $m i R-133 a$ levels was detected at 1 and 14 days post-anesthesia. The expression levels of $m i R-133 b$ and $m i R-206$, skeletal muscle-specific miRNAs, were increased significantly at 14 days post-anesthesia. These findings, in conjunction with the results presented above for the plasma muscle-specific miRNAs, indicate that the expression levels of muscle-specific miRNAs in cardiac and skeletal muscles and their plasma levels are substantially inversely correlated following sevoflurane anesthesia.

We also examined $m i R-21, m i R-24$ and $m i R-499$ in cardiac muscle (Fig. 3) as these miRNAs have been reported to protect cardiomyocytes against ischemia/reperfusion-induced apoptosis (14). A significant increase in $m i R-21$ and $m i R-499$ levels was detected at 3 and 7 days post-anesthesia (Fig. 3). The expression levels of $m i R-24$ increased significantly at 7 and 14 days post-anesthesia.

\section{Discussion}

In the present study, we examined the dynamics of circulating miRNAs as potentially informative markers for monitoring and assessing changes in miRNA expression due to sevoflurane anesthesia in organs and body systems. The main finding was that the majority of the circulating miRNAs in rat plasma were transiently downregulated as a result of sevoflurane anesthesia. With the exception of muscle-specific miRNAs (miR-1, $m i R-133 a, m i R-133 b$ and $m i R-206$ ), the levels of the plasma miRNAs which were downregulated increased substantially immediately after the anesthesia was terminated (in the recovery period). Furthermore, we revealed distinct plasma profiles of the muscle-specific miRNAs following sevoflurane anesthesia; the plasma levels of the muscle-specific miRNAs were persistently downregulated until 14 days post-anesthesia. Finally, we demonstrated that the expression levels of musclespecific miRNAs in cardiac and skeletal muscle and their plasma levels were substantially inversely correlated following sevoflurane anesthesia, suggesting that sevoflurane predominantly affects cardiac and skeletal muscle and suppresses the release of miRNAs from these tissues into the circulation. To the best of our knowledge, the present study is the first to demonstrate the circulating miRNA expression signature induced by sevoflurane anesthesia in rats.

Several miRNA genes are specifically expressed or highly enriched in cardiac and/or skeletal muscle, namely the muscle-specific miRNAs $(12,15)$. Muscle-specific miRNAs regulate the differentiation and proliferation of muscle cells (16). Recently, a number of studies have reported that miRNAs in plasma or serum are promising biomarkers for muscle diseases and myocardial injury (17-19). Mizuho et al (17) demonstrated that serum levels of $m i R-1$, $m i R-133 a$ and $m i R-206$ were increased in both the dystro- 
A

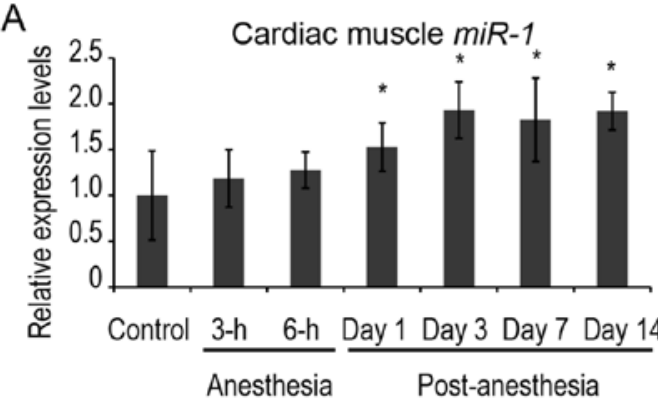

C

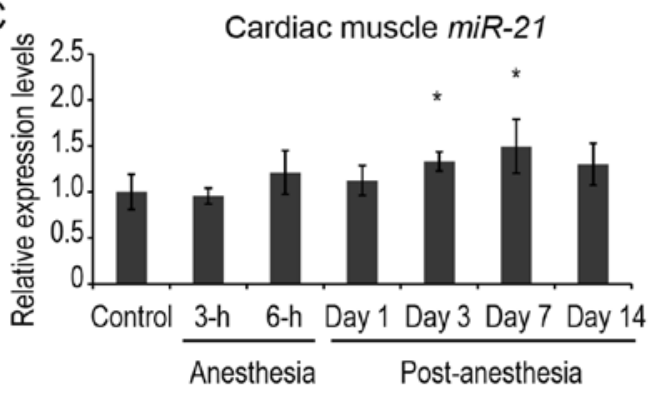

B

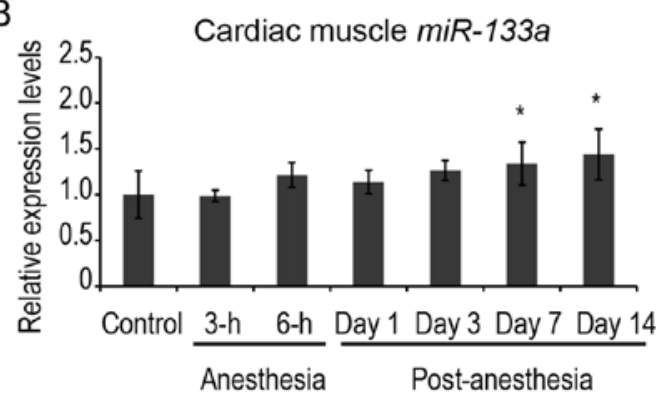

D

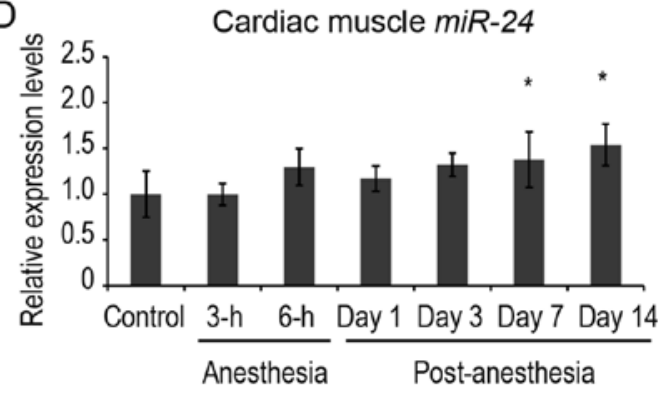

E

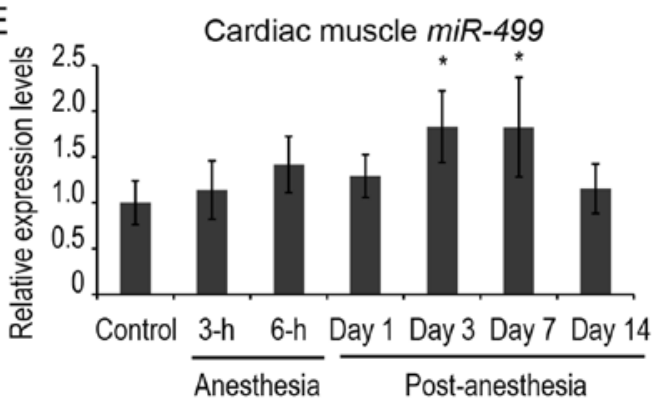

Figure 3. Expression levels of muscle-specific miRNAs in cardiac muscle. Expression levels of muscle-specific miRNAs [(A) $m i R-1$ and (B) $m i R-133 a$ ] and cardioprotective miRNAs [(C) $m i R-21$, (D) $m i R-24$ and (E) $m i R-499$ ] were examined by quantitative polymerase chain reaction (PCR). Time points for quantitative PCR analysis were: 3- and 6-h anesthesia, and at 1, 3, 7 and 14 days post-anesthesia. Data were normalized to Rnu6. Levels of miRNAs in the control group (no anesthesia) were defined as 1 . Data are the means \pm standard deviation (SD) (n=6 per group). Dunnett's test; ${ }^{*} \mathrm{P}<0.05$.

A

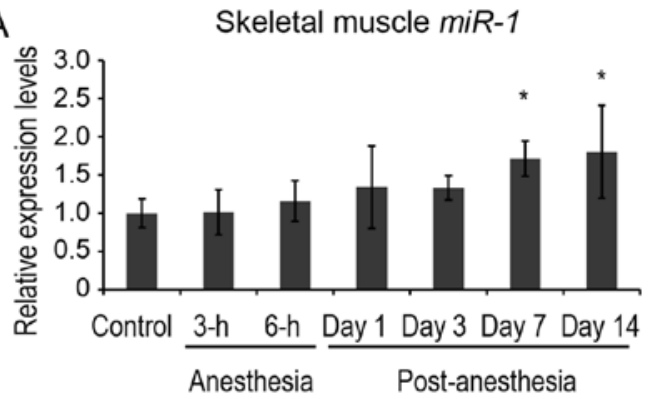

C

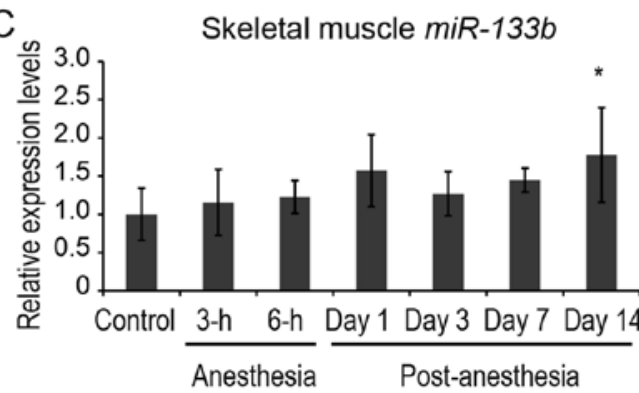

B

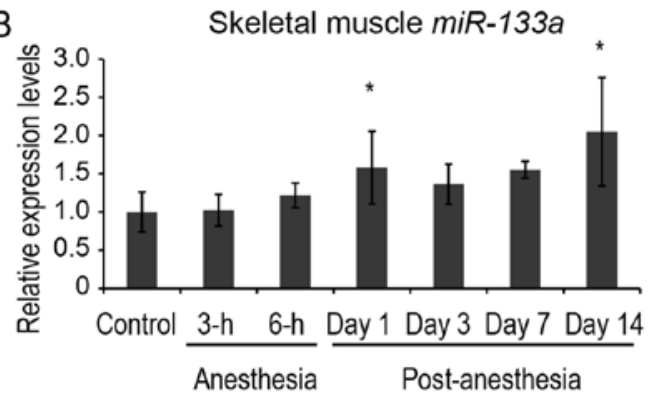

D

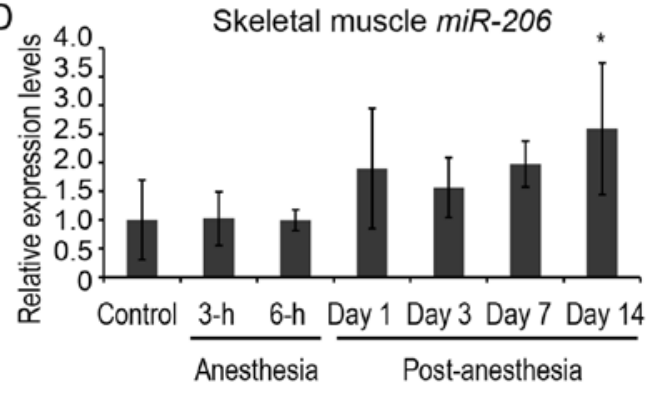

Figure 4. Expression levels of muscle-specific miRNAs in skeletal muscle. Expression levels of muscle-specific miRNAs [(A) $m i R-1$, (B) $m i R$-133a, (C) $m i R-133 b$ and (D) $m i R$-206] were examined by quantitative polymerase chain reaction (PCR). Time points for quantitative PCR analysis were: 3 - and 6-h anesthesia, and at 1,3,7 and 14 days post-anesthesia. Data were normalized to Rnu6. Levels of miRNAs in the control group (no anesthesia) were defined as 1 . Data are the means \pm standard deviation (SD) ( $\mathrm{n}=6$ per group). Dunnett's test; ${ }^{*} \mathrm{P}<0.05$. 
phin-deficient muscular dystrophy mouse model, mdx, and the canine X-linked muscular dystrophy in Japan (CXMDJ) dog model. Cheng et al (18) reported that in a rat model of acute myocardial infarction (AMI) induced by coronary ligation, serum levels of $m i R-1$ were increased significantly following AMI and that serum miR-1 levels with AMI showed a positive correlation with myocardial infarct size. The increase in circulating muscle-specific miRNAs in these muscle dystrophies and AMI models appears to be caused by an increase in the leakage of miRNAs from muscle tissues.

In the present study, we found that sevoflurane anesthesia significantly altered the plasma levels of muscle-specific miRNAs compared with the levels of other miRNAs. However, the mechanisms involved in this sevoflurane-induced downregulation of circulating muscle-specific miRNAs need to be elucidated. There are several possible explanations for these effects of sevoflurane. First, it is possible that the exosome-mediated secretion of miRNAs is attenuated in cardiac and skeletal muscle, resulting in the relatively lower plasma levels of muscle-specific miRNAs compared with those of other miRNAs. Another explanation may be that de novo miRNA synthesis is blocked in cardiac and skeletal muscle. To confirm whether the expression levels of muscle-specific miRNAs were altered by sevoflurane anesthesia in cardiac and skeletal muscle, we performed quantitative PCR analysis and found that the expression levels of muscle-specific miRNAs showed a negative correlation with the plasma levels (Figs. 3 and 4). Taking these findings into consideration, it seems likely that sevoflurane anesthesia affects miRNA secretion rather than de novo miRNA synthesis in cardiac and skeletal muscle. Circulating miRNAs are generally considered to be the result of exosomes that can protect circulating miRNAs from plasma RNase (8). However, a recent study raised an objection to this. Specifically, Roberts et al (20) demonstrated that the majority of serum muscle-specific miRNAs were protected from serum nucleases by the association with protein/lipoprotein complexes, rather than extracellular vesicles, in mdx mice. Thus, further studies are required in order to elucidate the molecular mechanisms of the sevoflurane-dependent muscle cell typespecific inhibition of miRNA secretion.

Sevoflurane exerts cardioprotective effects in anesthetic preconditioning (APC) when administered before a period of myocardial ischemia and reperfusion $(21,22)$. However, there have been few studies on the role of cardioprotective miRNAs in APC. Several myocardial ischemia-associated miRNAs have been reported to serve as cardioprotective miRNAs (14). For example, $m i R-21$ has been shown to be upregulated in the mouse heart following ischemic preconditioning (IPC) and sublethal heat shock, and exerts a protective effect on hypoxia/ reoxygenation-induced cell apoptosis, targeting programmed cell death 4 (23). IPC-induced miRNAs (miR-1, miR-21 and $m i R-24)$ exert cardioprotective effects similar to the delayed phase of IPC, possibly through upregulating endothelial nitric oxide synthase (eNOS), heat shock protein (HSP)70 and the HSP70 transcription factor, HSF-1 (24). miR-24 has been shown to suppress cardiomyocyte apoptosis, by repressing the gene encoding the $\mathrm{BH} 3$-only domain-containing protein 'Bim', which positively regulates apoptosis (25). miR-499 inhibits cardiomyocyte apoptosis through its suppression of the calcineurin-mediated dephosphorylation of dynamin-related protein-1 (26). In the present study, we investigated the effects of sevoflurane anesthesia on the expression levels of some cardioprotective miRNAs ( $m i R-21, m i R-24$ and $m i R-499)$ in cardiac muscle and found that a significant increase in their expression levels was also detected within 2 weeks post-anesthesia (Fig. 3). Sevoflurane may have cardioprotective effects by persistently upregulating endogenous miRNAs in cardiac muscle.

Unlike $m i R-133 b$ and $m i R-206, m i R-1$ and $m i R-133 a$, examined in this study, could be derived from cardiac and/or skeletal muscle $(12,15)$. $m i R-208$ and $m i R-499$ are primarily expressed in cardiac muscle (26); however, we did not determine significant changes in the levels of these miRNAs in the plasma. At present, the estimation of the proportion of circulating muscle-specific miRNAs derived from individual muscles remains an issue.

In conclusion, we demonstrted a circulating miRNA expression signature induced by sevoflurane anesthesia in rats. We revealed the persistent downregulation of muscle-specific miRNAs ( $m i R-1, m i R-133 a, m i R-133 b$ and $m i R-206)$ in rat plasma for 2 weeks following sevoflurane anesthesia. The expression levels of muscle-specific miRNAs in cardiac and skeletal muscle showed a negative correlation with their plasma levels. Our data suggest that sevoflurane anesthesia suppresses the release of miRNAs from cardiac and skeletal muscle tissue into the circulation, and this may contribute to the cardioprotective effects of sevoflurane. Although further functional and pathological studies on muscle-specific modification induced by sevoflurane are required, this new information provides novel insight towards a better understanding of the molecular mechanisms of action of the anesthetic, sevoflurane.

\section{Acknowledgements}

The authors are indebted to Mr. Takuji Kosuge (Department of Molecular Medicine and Anatomy, Nippon Medical School, Tokyo, Japan) for providing technical assistance. The present study was supported by Grants-in-Aid for Scientific Research from the Ministry of Education, Culture, Sports, Science and Technology (MEXT), Japan, and by a grant from the MEXT-Supported Program for the Strategic Research Foundation at Private Universities (2013-2017).

\section{References}

1. Brown BR Jr and Frink EJ: The safety of sevoflurane in humans. Anesthesiology 79: 201-202, 1993.

2. Obal D, Preckel B, Scharbatke H, Müllenheim J, Höterkes F, Thämer V and Schlack W: One MAC of sevoflurane provides protection against reperfusion injury in the rat heart in vivo. $\mathrm{Br}$ J Anaesth 87: 905-911, 2001.

3. Hu ZY and Liu J: Mechanism of cardiac preconditioning with volatile anaesthetics. Anaesth Intensive Care 37: 532-538, 2009.

4. Bartel DP: MicroRNAs: target recognition and regulatory functions. Cell 136: 215-233, 2009.

5. Le Quesne J and Caldas C: Micro-RNAs and breast cancer. Mol Oncol 4: 230-241, 2010

6. Small EM, Frost RJ and Olson EN: MicroRNAs add a new dimension to cardiovascular disease. Circulation 121: 1022-1032, 2010.

7. Weber JA, Baxter DH, Zhang S, Huang DY, Huang KH, Lee MJ, Galas DJ and Wang K: The microRNA spectrum in 12 body fluids. Clin Chem 56: 1733-1741, 2010.

8. Mitchell PS, Parkin RK, Kroh EM, Fritz BR, Wyman SK, Pogosova-Agadjanyan EL, Peterson A, Noteboom J, O'Briant KC, Allen A, Lin DW, Urban N, Drescher CW, Knudsen BS, Stirewalt DL, Gentleman R, Vessella RL, Nelson PS, Martin DB and Tewari M: Circulating microRNAs as stable blood-based markers for cancer detection. Proc Natl Acad Sci USA 105: 10513-10518, 2008. 
9. Tanaka S, Ishikawa M, Arai M, Genda Y and Sakamoto A: Changes in microRNA expression in rat lungs caused by sevoflurane anesthesia: a TaqMan ${ }^{\circledR}$ low-density array study. Biomed Res 33: 255-263, 2012.

10. Ishikawa M, Tanaka S, Arai M, Genda Y and Sakamoto A: Differences in microRNA changes of healthy rat liver between sevoflurane and propofol anesthesia. Anesthesiology 117: 1245-1252, 2012.

11. Orliaquet G, Vivien B, Langeron O, Bouhemad B, Coriat P and Riou B: Minimum alveolar concentration of volatile anesthetics in rats during postnatal maturation. Anesthesiology 95: 734-739, 2001

12. Liu N and Olson EN: MicroRNA regulatory networks in cardiovascular development. Dev Cell 18: 510-525, 2010.

13. Landgraf P, Rusu M, Sheridan R, Sewer A, Iovino N, Aravin A, Pfeffer S, Rice A, Kamphorst AO, Landthaler M, Lin C, Socci ND, Hermida L, Fulci V, Chiaretti S, Foà R, Schliwka J, Fuchs U, Novosel A, Müller RU, Schermer B, Bissels U, Inman J, Phan Q, Chien M, Weir DB, Choksi R, De Vita G, Frezzetti D, Trompeter HI, Hornung V, Teng G, Hartmann G, Palkovits M, Di Lauro R, Wernet P, Macino G, Rogler CE, Nagle JW, Ju J, Papavasiliou FN, Benzing T, Lichter P, Tam W, Brownstein MJ, Bosio A, Borkhardt A, Russo JJ, Sander C, Zavolan M and Tuschl T: A mammalian microRNA expression atlas based on small RNA library sequencing. Cell 129: 1401-1414, 2007.

14. Zhu H and Fan GC: Role of microRNAs in the reperfused myocardium towards post-infarct remodelling. Cardiovasc Res 94: 284-292, 2012.

15. Koutsoulidou A, Mastroyiannopoulos NP, Furling D, Uney JB and Phylactou LA: Expression of miR-1, miR-133a, miR-133b and miR-206 increases during development of human skeletal muscle. BMC Dev Biol 11: 34, 2011.

16. Townley-Tilson WH, Callis TE and Wang D: MicroRNAs 1, 133, and 206: critical factors of skeletal and cardiac muscle development, function, and disease. Int J Biochem Cell Biol 42 $1252-1255,2010$

17. Mizuho H, Nakamura A, Aoki Y, Ito N, Kishi S, Yamamoto K, Sekiguchi M, Takeda S and Hashido K: Identification of muscle-specific microRNAs in serum of muscular dystrophy animal models: promising novel blood-based markers for muscular dystrophy. PloS One 6: e18388, 2011.
18. Cheng Y, Tan N, Yang J, Liu X, Cao X, He P, Dong X, Qin S and Zhang C: A translational study of circulating cell-free microRNA-1 acute myocardial infarction. Clin Sci (Lond) 119: 87-95, 2010

19. Tijsen AJ, Pinto YM and Creemers EE: Circulating microRNAs as diagnostic biomarkers for cardiovascular diseases. Am J Physiol Heart Circ Physiol 303: H1085-H1095, 2012.

20. Roberts TC, Godfrey C, McClorey G, Vader P, Briggs D, Gardiner C, Aoki Y, Sargent I, Morgan JE and Wood MJ: Extracellular microRNAs are dynamic non-vesicular biomarkers of muscle turnover. Nucleic Acids Res 41: 9500-9513, 2013.

21. Toller WG, Kersten JR, Pagel PS, Hettrick DA and Warltier DC: Sevoflurane reduces myocardial infarct size and decreases the time threshold for ischemic preconditioning in dogs. Anesthesiology 91: 1437-1446, 1999.

22. Jakobsen CJ, Berg H, Hindsholm KB, Faddy N and Sloth E: The influence of propofol versus sevoflurane anesthesia on outcome in 10,535 cardiac surgical procedures. J Cardiothorac Vasc Anesth 21: 664-671, 2007

23. Cheng Y, Zhu P, Yang J, Liu X, Dong S, Wang X, Chun B, Zhuang $\mathbf{J}$ and Zhang $\mathrm{C}$ : Ischaemic preconditioning-regulated miR-21 protects heart against ischaemia/reperfusion injury via anti-apoptosis through its target PTCD4. Cardiovasc Res 87: 431-439, 2010.

24. Yin C, Salloum FN and Kukreja RC: A novel role of microRNA in late preconditioning: upregulation of endothelial nitric oxide synthase and heat shock protein 70. Circ Res 104: 572-575, 2009.

25. Qian L, Van Laake LW, Huang Y, Liu S, Wendland MF and Srivastava D: miR-24 inhibits apoptosis and represses Bim in mouse cardiomyocytes. J Exp Med 208: 549-560, 2011.

26. Wang JX, Jiao JQ, Li Q, Long B, Wang K, Liu JP, Li YR and Li PF: miR-499 regulates mitochondrial dynamics by targeting calcineurin and dynamin-related protein-1. Nat Med 17: 71-78, 2011. 